\title{
The efficiency of remote ischemic preconditioning on serum cystatin C-based acute kidney injury in patients undergoing coronary angiography; a randomized controlled trial
}

\author{
Azadeh Moradkhani ${ }^{1}{ }^{\mathbb{D}}$, Hamid Reza Samimagham ${ }^{2,3^{*}}{ }^{\mathbb{D}}$, Mohammad Tamaddondar $^{3}$, Hossein Farshidi², \\ Mahmood Khayatian ${ }^{4}$, Sanaz Soleimani ${ }^{1(\mathbb{D}}$ \\ ${ }^{1}$ Department of Internal Medicine, Shahid Mohammadi Hospital, Hormozgan University of Medical Sciences, Bandar Abbas, Iran \\ ${ }^{2}$ Cardiovascular Research Center, Hormozgan University of Medical Sciences, Bandar Abbas, Iran \\ ${ }^{3}$ Department of Nephrology and Internal Medicine, Shahid Mohammadi Hospital, Hormozgan University of Medical Sciences, Bandar \\ Abbas, Iran \\ ${ }^{4}$ Molecular Medicine Research Center and Department of Biochemistry, Faculty of Medicine, Hormozgan University of Medical Sciences, \\ Bandar Abbas, Iran
}

\section{AR T I C L E I N F O}

\section{Article Type:}

Original

\section{Article History:}

Received: 24 February 2020

Accepted: 6 June 2020

Published online: 24 July 2020

Keywords:

Contrast-induced nephropathy

Coronary angiography

serum cystatin $\mathrm{C}$

Acute kidney injury

Ischemic preconditioning

Preconditioning

\begin{abstract}
A B S T R A C T
Introduction: Contrast-induced acute kidney injury (CI-AKI) is a known complication of cardiac interventions. Remote ischemic preconditioning (RIPC) is a non-pharmacological method which has a nephroprotective effect. Serum cystatin $\mathrm{C}(\mathrm{Cys})$ is a suitable biomarker for the early diagnosis of AKI.

Objectives: This study aimed to evaluate the incidence of CI-AKI after RIPC in patients undergoing coronary angiography, through assessment of CysC.

Patients and Methods: Around 140 patients with stable coronary artery disease undergoing angiography were randomly allocated to two groups of RIPC and control groups. In each group, the following biomarkers were assessed: serum creatinine $(\mathrm{Cr})$ and $\mathrm{CysC}$ at baseline, 24-hour and 48-hour serum $\mathrm{Cr}$ and 24-hour CysC. The endpoint was the development of AKI based on either the KDIGO criteria or a $15 \%$ increase in serum CysC.

Results: No significant difference was observed between two groups regarding the incidence of AKI according to either KIDIGO criteria or by the increase of serum CysC $(P=0.116$ and $P=0.392$, respectively). Moreover, a $46.99 \%$ increase in CysC level was observed among patients with AKI during the first 24 hours after the procedure, while at the same interval, it increased only $16.01 \%$ in patients without AKI.

Conclusion: RIPC with three cycles of 5-minute ischemia and 5-minute reperfusion, did not decrease serum CysC based CI-AKI or alter renal biomarkers course in patients with low risk, who underwent coronary angiography.

Trial registration: This randomized controlled trial was registered in the Iranian registry of clinical trials (identifier: IRCT20180924041113N1; https://irct.ir/trial/34184, ethical code; HUMS. REC.1396.42).
\end{abstract}

Implication for health policy/practice/research/medical education:

According to our study, the three-cycle preconditioning approach did not decrease cystatin $\mathrm{C}(\mathrm{CysC})$ based contrast-induced acute kidney injury (CI-AKI) in 140 low-risk patients undergoing coronary angiography. Furthermore, CysC appears as a proper early biomarker for diagnosis of contrast-induced nephropathy, 24 hours after contrast media administration.

Please cite this paper as: Moradkhani A, Samimagham HR, Tamaddondar M, Farshidi H, Khayatian M, Soleimani S. The efficiency of remote ischemic preconditioning on serum cystatin C-based acute kidney injury in patients undergoing coronary angiography; a randomized controlled trial. J Nephropharmacol. 2021;10(1):09. DOI: 10.34172/npj.2021.09.

\section{Introduction}

Acute kidney injury (AKI) is a sudden renal failure within few hours to few days, causing the retention of toxic products that are normally excreted by the kidneys (1). AKI is a known complication after cardiac interventions since up to $30 \%$ of patients develop acute kidney damage 
after cardiac interventions; however, they rarely need dialysis (about 1\%) (2).

Contrast-induced acute kidney injury (CI-AKI) is a common cause of AKI in hospitalized patients, which is characterized by increased serum creatinine 48-72 hours after exposure to iodine-containing contrast medium. It might lead to longer hospitalization and intensive care unit (ICU) stay and a greater burden on the health care system (2).

In CI-AKI pathophysiology, either risk factors associated with the patients or risk factors associated with clinical interventions are involved (3). Moderate to severe renal dysfunction [glomerular filtration rate (GFR) $<60 \mathrm{~mL} / \mathrm{min}$ or serum creatinine $>1.36 \mathrm{mg} / \mathrm{dL}$ ] is the most important risk factor for progression towards CI-AKI followed by others including diabetes mellitus (DM), cardiac disorders, older patients, consumption of nephrotoxic drugs, contrast media administration with higher volume, reduced blood pressure during the surgery, and impaired hematocrit levels (due to dehydration or anemia) (4).

Several nephroprotective approaches have been described to address the increased incidence of contrastinduced nephropathy including using contrast medium with lower osmolality and preoperational hydrotherapy $(5,6)$. Considering the limitations and the complexity of the above-mentioned nephroprotective approaches, there is an ongoing need for an effective preventive strategy to avoid this complication (7).

The primary mechanism for the development of contrast-induced nephropathy seems to be the reperfusion injury. The prolonged renal hypoxia and ischemia and the following restoration of blood supply, resulting in the generating reactive oxygen species (ROS) and parenchymal damage and organ failure. An adaptive response has been described by several authors to improve the outcomes and protect against reperfusion injury called ischemic conditioning (4). Remote ischemic preconditioning (RIPC), is a protective intervention based on ischemic conditioning response. Although, it has shown promising results in reducing the incidence of CI-AKI and mortality associated with this complication, its long-term effect on kidney function is not clear (8). In fact, RIPC is a protective approach that, by creating shortterm impairment in non-vital organs, protects more vital organs against long-term ischemia (9).

Serum creatinine is the most common renal marker which is used for the diagnosis of AKI. Alongside its benefits, serum creatinine has several limitations mainly its inability for early renal dysfunction diagnosis. This led to numerous studies to find some biomarkers for early identification and treating of the CI-AKI (10). Among them, serum cystatin $\mathrm{C}(\mathrm{Cys} \mathrm{C})$ shows a promising result. CysC, a cysteine protease with low-molecular-weight, is stably developed by all of the nucleated cells, freely filtered by the glomeruli and fully reabsorbed in the proximal tubule without secretion (11).
CI-AKI can be independently predicted by serum concentration of CysC or its ratio to creatinine (12), while it has been suggested that changes in $\mathrm{Cys} C$ concentration can be a better biomarker for early diagnosis of CI-AKI in comparison to serum creatinine (13).

\section{Objectives}

In this study, the RIPC of arm with a three-cycle protocol and its effect on preventing CysC based CI-AKI following coronary angiography (CA) was investigated.

\section{Patients and Methods}

Study design and setting

This single-blinded randomized controlled trial (RCT) study was carried out at the heart clinic, Shahid Mohammadi hospital of the Hormozgan University of Medical Sciences (2017 to 2018). It investigates the effect of RIPC on AKI in patients receiving CA.

\section{Participants}

Around 140 patients undergoing CA were enrolled in this study. The inclusion criteria were patients aged $\geq 18$ years, with stable coronary artery disease requiring CA. Patients with renal dysfunction based on Kidney Disease Improving Global Outcomes (KDIGO) criteria (GFR $<60 \mathrm{cc} / \mathrm{min}$ ), pregnant women, emergency angiography, individuals with cardiogenic shock or recent cardiac infarction were excluded. The history and demographic information were taken from all patients. Informed consent was obtained from all participants.

\section{Intervention}

Seventy patients were assigned to either the treatment (RIPC) or the control group (no-RIPC) using random allocation software (Figure 1). A pneumatic cuff was wrapped around the upper arm one hour before the procedure. We chose the three-cycle method which was less frequently applied in previous studies. In both groups, three cycles of cuff inflation for 5 minutes followed by cuff deflation for another five minutes was performed. In the treatment group, the cuff was inflated to a pressure of 200 $\mathrm{mm} \mathrm{Hg}$ to induce transient limb ischemia. In the control group, sham RIPC was performed with the pneumatic cuff inflated to a non-occlusive $10 \mathrm{~mm} \mathrm{Hg}$. All participants underwent CA by an experienced cardiac interventionist who was blinded to patient group allocation.

Participants of either group received standard care. To achieve preferable clotting $300 \mathrm{mg}$ of aspirin and $300 \mathrm{mg}$ of clopidogrel was prescribed at least 6 hours before the CI with an additional administration of heparin (70-100 units $/ \mathrm{kg}$ ). The low-osmolality contrast medium volume was calculated based on the patient's weight. Nephrotoxic medications were withheld 72 hours before the procedure. The hydration plan included normal saline solution one $\mathrm{ml} / \mathrm{kg}$ of body weight per hour (reduced to $0.5 \mathrm{mg} / \mathrm{kg} / \mathrm{h}$ in patients with a left ventricular ejection fraction of $\leq 35 \%$ ) 


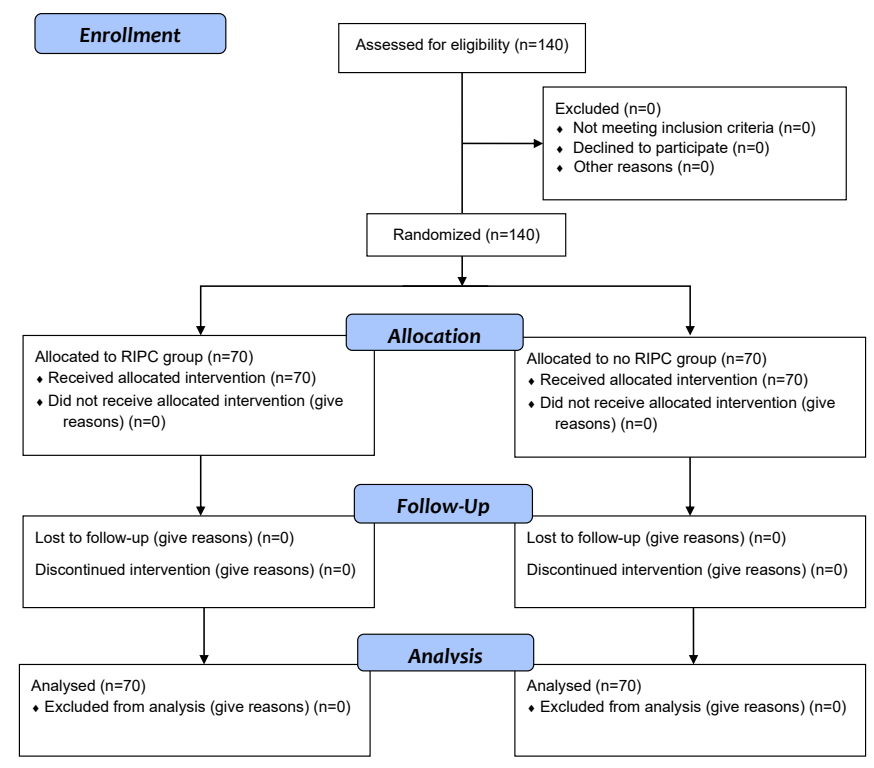

Figure 1. Consort flow diagram

for 12 hours before the procedure (whenever possible) and 24 hours after it.

\section{Study endpoints}

Blood samples were obtained before CA to measure serum creatinine $(\mathrm{Cr})$ and $\mathrm{Cys} C$ and estimated glomerular filtration rate (eGFR) [with CKD-EPI (the Chronic Kidney Disease Epidemiology Collaboration) formula]. After the procedure, 24-hour and 48-hour serum creatinine and eGFR and 24-hour CysC were measured. The primary study endpoint was acute renal failure development, detected by two criteria; the 2012 KDIGO AKI criteria (14) or a $15 \%$ increase in serum CysC [based on the paper published by Zhang et al in 2017 (15)].

The serum creatinine, eGFR, and serum $\mathrm{CysC}$ changes compared to the baseline values were evaluated and analyzed.

\section{Ethical issues}

This paper is a part of the thesis of Azadeh Moradkhani, in the internal medicine department of the Hormozgan University of Medical Sciences (HUMS). The study was conducted according to the Helsinki Declaration and informed consent was obtained from all participants. It was approved by the ethics board of HUMS (ethical code; HUMS.REC.1396.42) and registered in the Iranian registry of clinical trials (identifier: IRCT20180924041113N1; https://irct.ir/trial/34184). This research was supported by HUMS (Grant\# 960081).

\section{Statistical analysis}

The outcome measures were analyzed using the SPSS software version 22. The $t$ test and Mann-Whitney were used for the continuous variables with and without normal distribution, respectively. To evaluated categorical parameters, Pearson's chi-square and Fisher's exact test were used. The significance level was considered as $<0.05$.

Results

The patients' mean age was $56.3 \pm 11.8$ years and $56.8 \pm$ 11.4 years in the RIPC and control group, respectively $(P>0.05$; Table 1). Patients' detailed clinical data are shown in Table 2. As it is demonstrated, there was no significant difference between the two study groups regarding baseline characteristics.

\section{Clinical results}

Of 140 patients, 11 patients were diagnosed with CI-AKI by the KIDIGO, 8 in the RIPC group and 3 cases of AKI in the control group. Higher CI-AKI incidence in the RIPC group was not statistically significant $(P=0.116$; Table 3$)$.

When evaluating AKI with the CysC level, 59 patients were diagnosed, 27 in the RIPC group and 32 in the control group with no significant difference $(P=0.392$; Table 3).

No significant difference was observed regarding renal markers (i.e. serum creatinine, CysC, and eGFR) among the two study groups at baseline (Tables 5-7). In the RIPC

Table 1. Patients' age characteristic

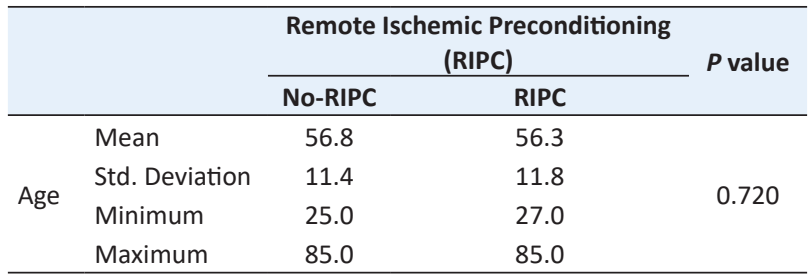


Table 2. Clinical characteristics of patients

\begin{tabular}{|c|c|c|c|c|c|c|}
\hline & & \multicolumn{4}{|c|}{ Remote Ischemic Preconditioning (RIPC) } & \multirow{3}{*}{$P$ value } \\
\hline & & \multicolumn{2}{|c|}{ No-RIPC } & \multicolumn{2}{|c|}{ RIPC } & \\
\hline & & No. & $\%$ & No. & $\%$ & \\
\hline \multirow{2}{*}{ Gender } & $\mathrm{F}$ & 32 & $45.7 \%$ & 29 & $41.4 \%$ & \multirow{2}{*}{0.609} \\
\hline & M & 38 & $54.3 \%$ & 41 & $58.6 \%$ & \\
\hline \multirow{2}{*}{ Smoking } & No & 50 & $71.4 \%$ & 47 & $67.1 \%$ & \multirow{2}{*}{0.583} \\
\hline & Yes & 20 & $28.6 \%$ & 23 & $32.9 \%$ & \\
\hline \multirow{2}{*}{ DM } & No & 53 & $75.7 \%$ & 57 & $81.4 \%$ & \multirow{2}{*}{0.410} \\
\hline & Yes & 17 & $24.3 \%$ & 13 & $18.6 \%$ & \\
\hline & & Mean & SD & Mean & SD & \\
\hline Contrast vo & & 0.664 & 0.506 & 0.614 & 0.400 & 0.529 \\
\hline \multirow{2}{*}{ Hematocrit } & Female & 38.2 & 3.6 & 39.2 & 3.7 & 0.290 \\
\hline & Male & 43.9 & 3.8 & 46.0 & 3.9 & 0.374 \\
\hline
\end{tabular}

Table 3. AKI occurrence by KIDIGO criteria

\begin{tabular}{|c|c|c|c|c|c|c|}
\hline & & \multicolumn{4}{|c|}{ Remote Ischemic Preconditioning (RIPC) } & \multirow{3}{*}{$P$ value } \\
\hline & & \multicolumn{2}{|c|}{ No-RIPC } & \multicolumn{2}{|c|}{ RIPC } & \\
\hline & & No. & $\%$ & No. & $\%$ & \\
\hline \multirow{2}{*}{ AKI } & No & 67 & $95.7 \%$ & 62 & $88.6 \%$ & \multirow{2}{*}{0.116} \\
\hline & Yes & 3 & $4.3 \%$ & 8 & $11.4 \%$ & \\
\hline
\end{tabular}

Table 4. CysC based AKI occurrence

\begin{tabular}{|c|c|c|c|c|c|c|}
\hline & & \multicolumn{4}{|c|}{ Remote Ischemic Preconditioning (RIPC) } & \multirow{3}{*}{$P$ value } \\
\hline & & \multicolumn{2}{|c|}{ No-RIPC } & \multicolumn{2}{|c|}{ RIPC } & \\
\hline & & No. & $\%$ & No. & $\%$ & \\
\hline \multirow{2}{*}{ AKI } & No & 38 & $54.3 \%$ & 43 & $61.4 \%$ & \multirow{2}{*}{0.392} \\
\hline & Yes & 32 & $45.7 \%$ & 27 & $38.6 \%$ & \\
\hline
\end{tabular}

Table 5. Serum creatinine changes

\begin{tabular}{|c|c|c|c|c|c|}
\hline & \multicolumn{4}{|c|}{ Remote Ischemic Preconditioning (RIPC) } & \multirow{3}{*}{$P$ value } \\
\hline & \multicolumn{2}{|c|}{ No-RIPC } & \multicolumn{2}{|c|}{ RIPC } & \\
\hline & Mean & Std. Deviation & Mean & Std. Deviation & \\
\hline Creatinine $0 \mathrm{~h}$ & 0.98 & 0.20 & 0.97 & 0.15 & $0.569 *$ \\
\hline Creatinine $24 \mathrm{~h}$ & 1.04 & 0.20 & 1.05 & 0.16 & $0.604 *$ \\
\hline Creatinine $48 \mathrm{~h}$ & 1.05 & 0.20 & 1.09 & 0.16 & $0.252 *$ \\
\hline$P$ value* & \multicolumn{2}{|c|}{$<0.001 * *$} & \multicolumn{2}{|c|}{$<0.001 * *$} & \\
\hline
\end{tabular}

* Independent-samples $t$ test; ** Friedman test.

Table 6. Estimated glomerular filtration rate (eGFR) changes

\begin{tabular}{|c|c|c|c|c|c|}
\hline & \multicolumn{4}{|c|}{ Remote Ischemic Preconditioning (RIPC) } & \multirow{3}{*}{$P$ value } \\
\hline & \multicolumn{2}{|c|}{ No-RIPC } & \multicolumn{2}{|c|}{ RIPC } & \\
\hline & Mean & Std. Deviation & Mean & Std. Deviation & \\
\hline GFR $0 \mathrm{~h}$ & 77.76 & 15.29 & 79.25 & 16.38 & $0.569 *$ \\
\hline GFR $24 \mathrm{~h}$ & 72.69 & 14.61 & 71.98 & 14.71 & $0.776 *$ \\
\hline GFR $48 \mathrm{~h}$ & 71.48 & 14.20 & 69.28 & 13.12 & $0.342 *$ \\
\hline$P$ value* & \multicolumn{2}{|c|}{$<0.001^{* *}$} & \multicolumn{2}{|c|}{$<0.001^{* *}$} & \\
\hline
\end{tabular}

* Independent-samples $t$ test; ${ }^{* *}$ Friedman test. 


\begin{tabular}{|c|c|c|c|c|c|}
\hline & \multicolumn{4}{|c|}{ Remote Ischemic Preconditioning (RIPC) } & \multirow{3}{*}{$P$ value } \\
\hline & \multicolumn{2}{|c|}{ No-RIPC } & \multicolumn{2}{|c|}{ RIPC } & \\
\hline & Mean & Std. Deviation & Mean & Std. Deviation & \\
\hline CysC O h & 20.81 & 11.11 & 20.41 & 10.43 & $0.814^{*}$ \\
\hline CysC $24 \mathrm{~h}$ & 25.20 & 16.02 & 23.80 & 12.46 & $0.509 *$ \\
\hline$P$ value* & \multicolumn{2}{|c|}{$<0.001^{* *}$} & \multicolumn{2}{|c|}{$<0.001^{* *}$} & \\
\hline
\end{tabular}

* Independent-samples $t$ test; ** Friedman test.

group, serum creatinine increased over time reaching $1.05 \mathrm{mg} / \mathrm{dL}$ and $1.09 \mathrm{mg} / \mathrm{dL}$ in the 24-hour and 48-hour evaluation, respectively compared to the baseline value $(0.97 \mathrm{mg} / \mathrm{dL})$. In the control group, serum creatinine also increased but with a lesser slope, raised from $0.98 \mathrm{mg} /$ $\mathrm{dL}$ at baseline to $1.04 \mathrm{mg} / \mathrm{dL}$ and $1.05 \mathrm{mg} / \mathrm{dL}$ in the $24-$ hour and 48-hour serum samples, respectively. As was demonstrated, the 24-hour and 48-hour serum creatinine and eGFR values were higher in the RIPC and control group, respectively with no significant difference (Tables 5 and 6). In either group, the ascending serum creatinine and descending eGFR course were statistically significant $(P<0.001)$.

According to Table 7, in the RIPC group serum CysC was $20.41 \mathrm{ng} / \mathrm{mL}$ and $23.81 \mathrm{ng} / \mathrm{mL}$, before the CA and 24 hours after, respectively. In the control group, the values were $20.81 \mathrm{ng} / \mathrm{mL}$ and $25.20 \mathrm{ng} / \mathrm{mL}$, respectively. As it is shown, serum CysC was lower in the RIPC group, with no significant differences. In either group, the ascending course of the serum CysC was statistically significant $(P$ $<0.001)$.

In patients with CI-AKI, the 24 -hour and 48 -hour serum creatinine increased by $28.5 \%$ and $40.4 \%$, respectively $(1.08 \mathrm{mg} / \mathrm{dL}$ and $1.18 \mathrm{mg} / \mathrm{dL}$ compared to $0.84 \mathrm{mg} /$ $\mathrm{dL}$ ), while in other patients, this renal marker increased $6.1 \%$ and $8.1 \%$, respectively $(1.04 \mathrm{mg} / \mathrm{dL}$ and $1.06 \mathrm{mg} /$ $\mathrm{dL}$ compared to $0.98 \mathrm{mg} / \mathrm{dL}$ ) (Figure 2).The eGFR course was similar to serum creatinine. In AKI patients, eGFR decreased to $70.36 \mathrm{~mL} / \mathrm{min}$ and $61.94 \mathrm{~mL} / \mathrm{min}$ after 24

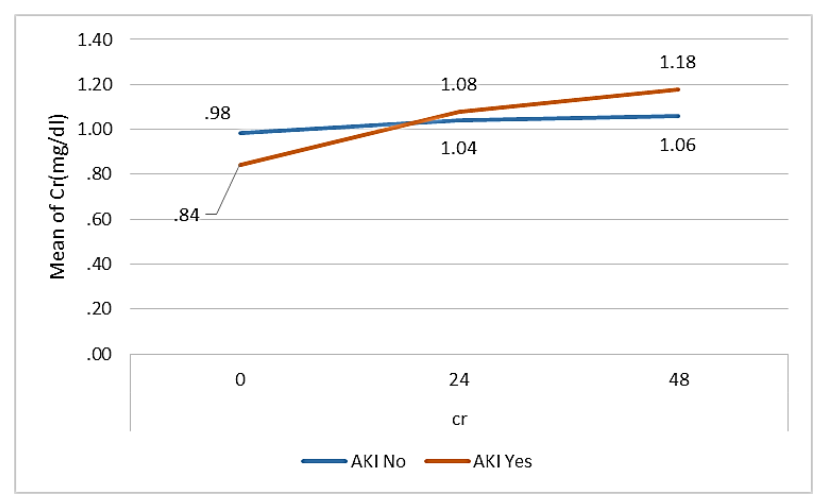

Figure 2. Comparison of serum creatinine in patients with and without AKI hours and 48 hours after the CA, from the baseline value of $88.48 \mathrm{~mL} / \mathrm{min}$, whereas in patients without AKI, it reduced from $77.66 \mathrm{~mL} / \mathrm{min}$ to $72.51 \mathrm{~mL} / \mathrm{min}$ and 71.10 $\mathrm{mL} / \mathrm{min}$, respectively (Figure 3).

A significant serum $\mathrm{Cys} C$ increase was observed over the first 24 hours of the $\mathrm{CI}$ in patients with CI-AKI. The serum CysC values increased to the $45.05 \%$ of the baseline values (from $25.77 \mathrm{ng} / \mathrm{mL}$ to $37.88 \mathrm{ng} / \mathrm{mL}$ ), while at the same time, in patients without CI-AKI, it increased by $16.01 \%$ (20.17 ng/mL to $23.40 \mathrm{ng} / \mathrm{mL}$; Figure 4$)$.

\section{Discussion}

CI-AKI is recognized as one of the common complications of coronary angiography (16). The growing need for contrast mediums resulted in the increasing incidence of this complication which has become the third most

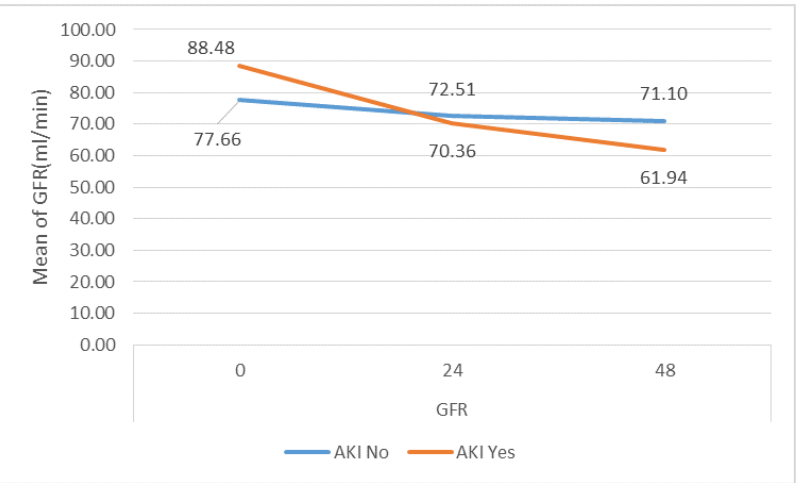

Figure 3. Comparison of eGFR in patients with and without AKI.

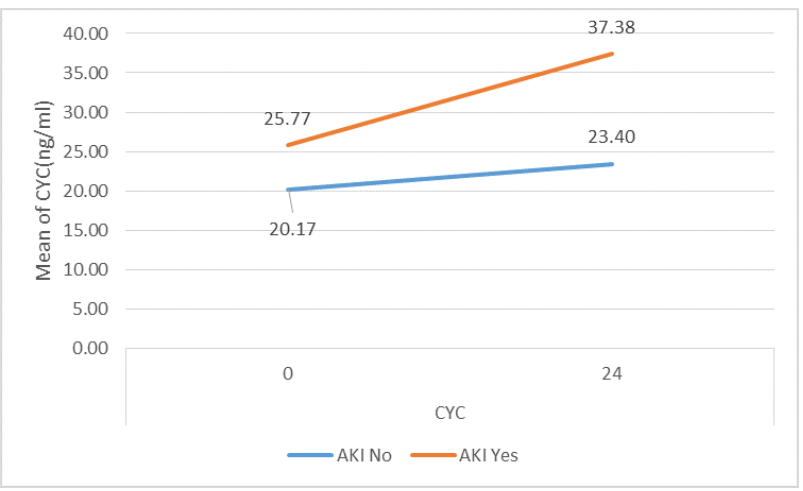

Figure 4. Comparison of serum CysC in patients with and without AKI. 
common cause of renal failure (17). The risk of dialysis after CI-AKI is generally less than $1 \%$ but may increase to $5-50 \%$ when accompanying certain morbidities including patients with a chronic renal problem, DM and high blood pressure (18).

Due to the shortcoming of usual nephroprotective approaches, there is a growing debate to introduce an effective approach to protect patients against contrastinduced nephropathy $(6,7)$. Base on this necessity, an intervention has been described consisting of several transient non-lethal ischemia cycles called remote ischemic conditioning (RIC) (9). One of the most common approaches of RIC is the RIPC method which we used in the present study (19). Several authors in their studies showed the renoprotective of RIPC during surgeries (20). In this study, we investigated the effect of 3-cycle RIPC of the upper arm on preventing CysC based CI-AKI following coronary angiography. The principal finding of this study was that RIPC with the above-mentioned method, neither decrease CI-AKI occurrence nor alter serum creatinine, CysC and eGFR course after coronary angiography.

Several RCTs investigated the role of the RIPC in patients undergoing cardiac surgery and coronary angiography with good results concerning the incidence of AKI $(2,6,8,21,22)$. However, other studies failed to demonstrate its protective effects $(23,24)$. Several metaanalysis also failed to reach a consistent conclusion, since the meta-analysis by Li et al (25) and also by Yasin et al (26) indicated that RIPC had no protective effect on CI-AKI. However, these meta-analysis included participants who had undergone different interventions, such as cardiac or vascular surgery, complex valvular heart surgery, complex congenital heart disease surgery, or percutaneous coronary intervention. On the contrary, several other meta-analyses showed that RIPC decreased the risk of acute renal failure and supported the kidney protective role of this method (7,27-29).

These conflicting findings could be attributed to the different methods used for RIPC. Using three- or fourcycle approach in upper or lower extremities were the most common methods used to induce this intervention, respectively. In our study, we performed 3-cycle, 5-minute RIPC applied to the arm. Choi et al (23) performed the 3-cycle RIPC, but with 10-minute cycles, and in the lower extremities in patients undergoing complex valvular heart surgery. According to their study, although, RIPC failed to reduce the renal injury, it was associated with better myocardial function and less ICU stay. In Zhou et al meta-analysis study (7), subgroup analysis revealed that RIC induced through 4 cycle protocol, had a significant renoprotective role compared to other protocols. Along with the findings of this study, Dong et al (30) and Lu et al (31) confirmed the relation between conditioning cycles and their efficiency. They demonstrated that RIC with more cycles is associated with a better response.
Er et al (21) and Igarashi et al (22), both performed preconditioning with a 4-cycle protocol in high risk and low-moderate risk patients undergoing elective coronary angiography, respectively and found the RIPC to be preventive of CI-AKI with the mentioned protocol. The 3-cycle approach used by Zarbock et al (2) was successfully effective in reducing AKI occurrence during cardiac surgery only among high-risk patients. Their findings were contrary to our findings which failed to demonstrate the nephroprotective effect of the RIPC. In comparison, we excluded patients with renal dysfunction (GFR $<60 \mathrm{cc} /$ min), but in the study of Zarbock et al, only patients with high risk for AKI were included. Based on these findings, we proposed that the protective role of the RIPC is seen only in the high-risk population (32). In addition, we studied CI-AKI occurrence after coronary angiography, whereas Zarbock et al focused on patients undergoing cardiac surgery with the use of cardiopulmonary bypass.

Accordingly, Cheung et al (33) performed RIPC with 4 cycles of 5-minute ischemia by inflating a cuff around a leg and found evidence of neither the renal protective role of RIPC nor its association with renal biomarkers' improvement, a finding similar to our study. Rear et al (24) investigated whether 4 cycles RIPC could safely reduce the incidence of CI-AKI in patients with impaired renal function undergoing elective or emergency coronary angiography or PCI? No difference in CI-AKI occurrence or secondary outcome (serum creatinine and urine albumin to creatinine ratio) measures was demonstrated between RIPC and control group. Despite the neutral outcome of the study, several hypothesis-forming findings were uncovered, including whether RIPC might be more efficacious in patients at very high-risk of CI-AKI or in participants with DM who may not have been responsive to the RIPC protocol. In our study, RIPC was not effective in patients at low-risk of AKI.

Furthermore, we noted that RIPC did not change the course of renal biomarkers after coronary angiography. According to either Li et al (28) or Yang et al (29), RIPC was not associated with a significant difference in the course of GFR and serum creatinine after surgery. Choi et al (23), reported no significant differences in serum levels of creatinine, CysC, and NGAL between groups throughout the study period. Plausible causes for the observations of this result may be that RIPC is more effective in protecting organs enduring direct and obvious ischemia-reperfusion insult, resulting in organ damage associated with marked pro-inflammatory and oxidative stress. Hence, RIPC has shown its renal protective effects in major vascular surgery or interventions without perfusion to the kidneys (32).

Generally, the serum creatinine elevation compared to the base level is used as the gold standard for the diagnosis of AKI (34). However, the detection of CI-AKI using serum creatinine, led to the prolonged duration of hospitalization in most patients due to its late elevation in response to renal dysfunction (35). In addition, serum 
creatinine has a low-sensitivity in some cases of acute dysfunction, as it may only increase when the renal function decrease to $50 \%$ (36). In addition, this biomarker is affected by several renal and non-renal factors such as muscle mass changes, tubular secretion, race, age and gender (37). Due to these problems, recent studies have looked at other biomarkers such as neutrophil gelatinaseassociated lipocalin (NGAL) and CysC for the diagnosis of AKI. However, their results, in particular, their role in early detection of AKI, have conflicting results (13). Of these biomarkers, CysC is filtered in the glomeruli freely, since nearly all of it, reabsorbed in the proximal tubule (38). The life cycle of this biomarker is also approximately half that of the creatinine, resulting in an earlier change compared to serum creatinine (39). As a result, it is a good candidate for the early diagnosis of CI-AKI (34). In several meta-analysis studies, the predicting value of this biomarker in comparison with creatinine serum has been reported (40).

We used two criteria for diagnosing AKI. Only 11 patients were diagnosed to have AKI when using KIDIGO criteria, but 59 cases were detected when using a $15 \%$ increase in serum CysC as the diagnosing method. In the study by Zhang et al (15), serum CysC is a more sensitive marker than serum creatinine in identifying CI-AKI cases. The disparity between serum CysC and serum creatinine in CI-AKI detection might be because of the properties of CysC, including shorter half-life, absence of renal tubular secretion, and less affected by volume status (11). Furthermore, in the present study, in patients with AKI in the first 24 hours, serum CysC increased roughly twice the rate of serum creatinine ( $45 \%$ versus $28.5 \%$ ), since, CysC elevation in this period, was more significant than the 48 hour serum creatinine increment (45\% versus $40.4 \%$ ). As a result, it can be used as an appropriate biomarker for earlier CI-AKI detection.

In the study by Yong study et al (41), serum CysC was a valuable and powerful marker for the diagnosis of AKI with different causes. The detailed assessment parameters, such as sensitivity, specificity, positive likelihood ratio, negative likelihood ratio, and diagnostic odds ratio for $\mathrm{CysC}$ were $0.82,0.82,4.6,0.22$ and 21, respectively. CysC performed the best accuracy in CI-AKI. The probable reason might be that kidney injury and hemodynamic disorder induced by CI-AKI is less serious and complicated than that by other AKI causes. Several novel biomarkers have been described to diagnose CI-AKI. Of them, NGAL, interleukin-18 (IL18) and CysC are the most promising (42). However, the former two biomarkers lack established determination method in clinical laboratories. Thus, according to the results of the study of Yong et al (41), CysC could be the optimal marker predicting various AKI.

Kim et al (43), found elevated baseline CysC levels to be an independent risk factor for CI-AKI and is a predictor of all-cause mortality and major adverse events in patients with peripheral artery disease undergoing endovascular therapy. Accordingly, Shukla et al (35) conclude that a rise of $\geq 10 \%$ in serum CysC at 24 hours could be used as a reliable marker for the identification of CI-AKI.

In some studies, the $\mathrm{CysC}$ role was compared to other biomarkers. Fu et al (34) suggested that comparing the $\mathrm{Cys} \mathrm{C}$ and serum creatinine, $\mathrm{Cys} \mathrm{C}$ was a more reliable marker of early diagnosis of contrast-induced nephropathy. Wacker-Gußmann et al (12) demonstrated that CI-AKI in patients with creatinine within the upper normal range (between 0.8 and $1.3 \mathrm{mg} / \mathrm{dL}$ ) is significantly more frequent after intra-arterial than after intravenous contrast administration. In these patients, renal impairment after contrast administration is independently predicted by CysC and CysC/creatinine-ratio, whereas blood urea nitrogen (BUN) and creatinine were not predictive. Liu et al (44) noted that elevated CysClevel is a reliable marker for $\mathrm{CI}-\mathrm{AKI}$; however, the predictive value of CysC-based CIAKI varies with the definition of serum creatinine-based CI-AKI, without good prediction of long-term outcomes. In the study of Cecchi et al (45), neither baseline serum and urinary NGAL nor their variations after percutaneous coronary invasive procedures (PCIP) were related to CIAKI occurrence, while CysC results were associated with CI-AKI development, earlier than creatinine and eGFR variations. On the contrary, in the study by $\mathrm{Xu}$ et al (46), serum CysC was not a better marker to evaluate CI-AKI after angiography compared to serum creatinine. In our study, it should be noted that serum creatinine and CysC measurement were obtained 48 hours and 72 hours after intervention since blood samples were not taken at 12 or 24 hours of surgery to assess the diagnostic power of CysC and serum creatinine for early detection of CI-AKI.

Despite the strength of this study which was the first to evaluate the effect of RIPC on the CysC based CI-AKI, it has several limitations that should be addressed in further studies. Some of these limitations were small sample size, evaluation of short-term renal function and investigating only in low-risk patients.

\section{Conclusion}

Our study demonstrated that RIPC, with three cycles of 5-minute ischemia and 5-minute reperfusion, did not decrease serum CysC based CI-AKI or alter renal biomarkers course in patients with low risk undergoing coronary angiography. Serum CysC is an appropriate biomarker for early diagnosis of CI-AKI, 24 hours after receiving the contrast.

\section{Study limitations}

This study has several limitations that should be addressed in further studies. The main limitations were small sample size, evaluation of short-term renal function and investigating RIPC only in low-risk patients. 


\section{Authors' contribution}

HS designed the study, observed the accuracy and validity of the study. AM collected the data. MA analyzed data. MT supervised the project. AM wrote the paper. All authors edited and revised the final manuscript.

\section{Conflicts of interest}

The authors declare no conflict of interest regarding the publication of this article.

\section{Ethical considerations}

Ethical issues including plagiarism, double publication, and redundancy have been completely observed by the authors.

\section{Funding/Support}

This research was supported by Hormozgan University of Medical Sciences (Grant\# 960081).

\section{References}

1. Basile DP, Anderson MD, Sutton TA. Pathophysiology of acute kidney injury. Compr Physiol. 2012;2:1303-53. doi: 10.1002/cphy.c110041.

2. Zarbock A, Schmidt C, Van Aken H, Wempe C, Martens S, Zahn PK, et al. Effect of remote ischemic preconditioning on kidney injury among high-risk patients undergoing cardiac surgery: a randomized clinical trial. JAMA. 2015;313:213341. doi: 10.1001/jama.2015.4189.

3. Azzalini L, Spagnoli V, Ly HQ. Contrast-induced nephropathy: from pathophysiology to preventive strategies. Can J Cardiol. 2016;32:247-55. doi: 10.1016/j. cjca.2015.05.013.

4. Tapuria N, Kumar Y, Habib MM, Amara MA, Seifalian AM, Davidson BR. Remote ischemic preconditioning: a novel protective method from ischemia reperfusion injury-a review. J Surg Res. 2008;150:304-30. doi: 10.1016/j.jss.2007.

5. Deftereos S, Giannopoulos G, Tzalamouras V, Raisakis K, Kossyvakis C, Kaoukis A, et al. Renoprotective effect of remote ischemic post-conditioning by intermittent balloon inflations in patients undergoing percutaneous coronary intervention. J Am Coll Cardiol. 2013;61:1949-55. doi: 10.1016/j.jacc.2013.02.023.

6. Chen SL, Zhang J, Yei F, Zhu Z, Liu Z, Lin S, et al. Clinical outcomes of contrast-induced nephropathy in patients undergoing percutaneous coronary intervention: a prospective, multicenter, randomized study to analyze the effect of hydration and acetylcysteine. Int J Cardiol. 2008;126:407-13. doi: 10.1016/j.ijcard.2007.05.004.

7. Zhou CC, Yao WT, Ge YZ, Xu LW, Wu R, Gao XF, et al. Remote ischemic conditioning for the prevention of contrast-induced acute kidney injury in patients undergoing intravascular contrast administration: a meta-analysis and trial sequential analysis of 16 randomized controlled trials. Oncotarget. 2017;8:79323-36. doi: 10.18632/ oncotarget.18106.

8. Bell RM, Rear R, Cunningham J, Dawnay A, Yellon DM. Effect of remote ischaemic conditioning on contrastinduced nephropathy in patients undergoing elective coronary angiography (ERICCIN): rationale and study design of a randomised single-centre, double-blind placebo- controlled trial. Clin Res Cardiol. 2014;103:203-209. doi: 10.1007/s00392-013-0637-3.

9. Meybohm P, Bein B, Brosteanu O, Cremer J, Gruenewald $\mathrm{M}$, Stoppe C, et al. A multicenter trial of remote ischemic preconditioning for heart surgery. $\mathrm{N}$ Engl J Med. 2015;373:1397-407. doi: 10.1056/NEJMoa1413579.

10. Shaker O, El-Shehaby A, El-Khatib M. Early diagnostic markers for contrast nephropathy in patients undergoing coronary angiography. Angiology. 2010;61:731-6. doi: 10.1177/0003319710373093.

11. Soto K, Coelho S, Rodrigues B, Martins H, Frade F, Lopes S, Cunha L, Papoila AL, Devarajan P. Cystatin C as a marker of acute kidney injury in the emergency department. Clin J Am Soc Nephrol. 2010;5:1745-54. doi: 10.2215/CJN.00690110

12. Wacker-Gußmann A, Bühren K, Schultheiss C, Braun SL, Page S, Saugel B, et al. Prediction of contrast-induced nephropathy in patients with serum creatinine levels in the upper normal range by cystatin C: a prospective study in 374 patients. AJR Am J Roentgenol. 2014;202:452-8. doi: 10.2214/AJR.13.10688.

13. Wang M, Zhang L, Yue R, You G, Zeng R. Significance of xystatinCforearlydiagnosis of contrast-inducednephropathy in patients undergoing coronary angiography. Med Sci Monit. 2016;22:2956-61. doi:10.12659/MSM.897241

14. Kellum JA, Lameire N, Aspelin P, Barsoum RS, Burdmann EA, Goldstein SL, et al. Kidney disease: improving global outcomes (KDIGO) acute kidney injury work group. KDIGO clinical practice guideline for acute kidney injury. Kidney Int Suppl. 2012;2:1-138. doi: 10.1038/kisup.2012.1

15. Zhang WF, Zhang T, Ding D, Sun SQ, Wang XL, Chu SC, et al. Use of both serum cystatin $\mathrm{c}$ and creatinine as diagnostic criteria for contrast-induced acute kidney injury and its clinical implications. J Am Heart Assoc. 2017;6:e004747. P doi:10.1161/JAHA.116.004747

16. Bhandari P, Shah Z, Patel K, Patel R. Contrast-induced acute kidney injury following coronary angiography in patients with end-stage liver disease. J Community Hosp Intern Med Perspect. 2019;9:403-409. doi:10.1080/20009666.2019.1661 148

17. Nash K, Hafeez A, Hou S. Hospital-acquired renal insufficiency. Am J Kidney Dis. 2002;39:930-6. doi: 10.1053/ ajkd.2002.32766.

18. McCullough PA. Contrast-induced acute kidney injury. J Am Coll Cardiol. 2008;51:1419-28. doi: 10.1016/j. jacc.2007.12.035.

19. Cao B, Zhang C, Wang H, Xia M, Yang X. Renoprotective effect of remote ischemic postconditioning in patients with ST-elevation myocardial infarction undergoing primary percutaneous coronary intervention. Ther Clin Risk Manag. 2018;14:369-375. doi: 10.2147/TCRM.S158768

20. Candilio L, Malik A, Ariti C, Barnard M, Di Salvo C, Lawrence $\mathrm{D}$, et al. Effect of remote ischaemic preconditioning on clinical outcomes in patients undergoing cardiac bypass surgery: a randomised controlled clinical trial. Heart. 2015;101:185-92. doi: 10.1136/heartjnl-2014-306178.

21. Er F, Nia AM, Dopp H, Hellmich M, Dahlem KM, Caglayan $\mathrm{E}$, et al. Ischemic preconditioning for prevention of contrast medium-induced nephropathy randomized pilot RenPro trial (renal protection trial). Circulation. 2012;126(3):296303. doi: 10.1016/j.jvs.2012.12.013.

22. Igarashi $\mathrm{G}$, Iino $\mathrm{K}$, Watanabe $\mathrm{H}$, Ito $\mathrm{H}$. Remote ischemic pre-conditioning alleviates contrastinduced acute kidney injury in patients with moderate chronic kidney disease. 
Circ J. 2013;77:3037-44.

23. Choi YS, Shim JK, Kim JC, Kang KS, Seo YH, Ahn KR, Kwak YL. Effect of remote ischemic preconditioning on renal dysfunction after complex valvular heart surgery: a randomized controlled trial. J Thorac Cardiovasc Surg. 2011;142:148-54. doi: 10.1016/j.jtcvs.2010.11.018.

24. Rear R. The effect of remote ischaemic preconditioning on contrast induced nephropathy in the clinical setting of coronary angiography and percutaneous coronary intervention [dissertation]. London: UCL University College; 2017.

25. Li L, Li G, Yu C, Li Y. The role of remote ischemic preconditioning on postoperative kidney injury in patients undergoing cardiac and vascular interventions: a metaanalysis. J Cardiothorac Surg. 2013;8:43. doi:10.1186/17498090-8-43

26. Yasin NA, Herbison P, Saxena P, Praporski S, Konstantinov IE. The role of remote ischemic preconditioning in organ protection after cardiac surgery: a meta-analysis. J Surg Res. 2014;186:207-16. doi: 10.1016/j.jss.2013.09.006.

27. Bei W-j, Duan C-y, Chen J-y, Wang K, Liu Y-h, Liu Y, et al. Remote ischemic conditioning for preventing contrast-induced acute kidney injury in patients undergoing percutaneous coronary interventions/coronary angiography: a meta-analysis of randomized controlled trials. J Cardiovasc Pharmacol Ther. 2016;21:53-63. doi: $10.1177 / 1074248415590197$.

28. Li B, Lang X, Cao L, Wang Y, Lu Y, Feng S, et al. Effect of remote ischemic preconditioning on postoperative acute kidney injury among patients undergoing cardiac and vascular interventions: a meta-analysis. J Nephrol. 2017;30:19-33. doi: 10.1007/s40620-016-0301-x.

29. Yang Y, Lang XB, Zhang P, Lv R, Wang YF, Chen JH. Remote ischemic preconditioning for prevention of acute kidney injury: a meta-analysis of randomized controlled trials. Am J Kidney Dis. 2014;64:574-83. doi: 10.1053/j. ajkd.2014.04.029.

30. Dong S, Cao Y, Li H, Tian J, Yi C, Sang W. Impact of ischemic preconditioning on ischemia-reperfusion injury of the rat sciatic nerve. Int J Clin Exp Med. 2015;8:16245-16251.

31. Lu Y, Dong C-s, Yu J-m, Li H. Morphine reduces the threshold of remote ischemic preconditioning against myocardial ischemia and reperfusion injury in rats: the role of opioid receptors. J Cardiothorac Vasc Anesth. 2012;26:403-6. doi: 10.1053/j.jvca.2011.07.036.

32. Hausenloy DJ, Bøtker HE, Condorelli G, Ferdinandy P, Garcia-Dorado D, Heusch G, et al. Translating cardioprotection for patient benefit: position paper from the Working Group of Cellular Biology of the Heart of the European Society of Cardiology. Cardiovasc Res. 2013;98:727. doi: $10.1093 / \mathrm{cvr} / \mathrm{cvt} 004$.

33. Cheung MM, Kharbanda RK, Konstantinov IE, Shimizu M, Frndova H, Li J, et al. Randomized controlled trial of the effects of remote ischemic preconditioning on children undergoing cardiac surgery: first clinical application in humans. J Am Coll Cardiol. 2006;47:2277-82. doi: 10.1016/j. jacc.2006.01.066.
34. Fu N, Liang M, Yang S. High loading dose of atorvastatin for the prevention of serum creatinine and cystatin c-based contrast-induced nephropathy following percutaneous coronary intervention. Angiology. 2018:0003319717750903. doi: 10.1177/0003319717750903.

35. Shukla AN, Juneja M, Patel H, Shah KH, Konat A, Thakkar BM, et al. Diagnostic accuracy of serum cystatin C for early recognition of contrast induced nephropathy in Western Indians undergoing cardiac catheterization. Indian Heart J. 2017;69:311-5. doi: 10.1016/j.ihj.2016.12.010.

36. Thomas ME, Blaine C, Dawnay A, Devonald MA, Ftouh S, Laing C, et al. The definition of acute kidney injury and its use in practice. Kidney Int. 2015;87:62-73. doi: 10.1038/ ki.2014.328.

37. Parikh CR, Devarajan P. New biomarkers of acute kidney injury. Crit Care Med. 2008;36:S159-65. doi: 10.1097/ CCM.0b013e318168c652.

38. Gowda S, Desai PB, Kulkarni SS, Hull VV, Math AA, Vernekar SN. Markers of renal function tests. N Am J Med Sci. 2010;2:170-3.

39. Odutayo A, Cherney D. Cystatin C and acute changes in glomerular filtration rate. Clin Nephrol. 2012;78:64-75.

40. Zhang Z, Lu B, Sheng X, Jin N. Cystatin C in prediction of acute kidney injury: a systemic review and metaanalysis. Am J Kidney Dis. 2011;58:356-65. doi: 10.1053/j. ajkd.2011.02.389.

41. Yong Z, Pei X, Zhu B, Yuan H, Zhao W. Predictive value of serum cystatin $C$ for acute kidney injury in adults: a metaanalysis of prospective cohort trials. Sci Rep. 2017;7:41012. doi:10.1038/srep41012

42. Connolly M, McEneaney D, Menown I, Morgan N, Harbinson M. Novel biomarkers of acute kidney injury after contrast coronary angiography. Cardiol Rev. 2015; 23:240-6. doi: $10.1097 /$ CRD.0000000000000058.

43. Kim GS, Ko YG, Shin DH, Kim JS, Kim BK, Choi D, et al. Elevated serum cystatin $C$ level is an independent predictor of contrast-induced nephropathy and adverse outcomes in patients with peripheral artery disease undergoing endovascular therapy. J Vasc Surg. 2015;61:1223-30. doi: 10.1016/j.jvs.2014.11.079.

44. Liu Y, Li H, Li LW, Tan N, Chen S. Cystatin c- and creatininebased definitions of contrast-induced nephropathy after coronary angiography or percutaneous coronary intervention. J Am Coll Cardiol. 2016;67:330. doi: 10.1016/ S0735-1097(16)30331-X

45. Cecchi E, Avveduto G, D’Alfonso MG, Terreni A, Gelera E, Caldini A, Giglioli C. Cystatin C, but not urinary or serum NGAL, may be associated with contrast induced nephropathy after percutaneous coronary invasive procedures: a single center experience on a limited number of patients. Acta Med Acad. 2017;46:34-43. doi: 10.5644/ ama2006-124.184.

46. Xu Q, Wang NN, Duan SB, Liu N, Lei R, Cheng W, Zhou SK. Serum cystatin $\mathrm{c}$ is not superior to serum creatinine for early diagnosis of contrast-induced nephropathy in patients who underwent angiography. J Clin Lab Anal. 2017;31: e22096. doi: 10.1002/jcla.22096.

Copyright $\odot 2021$ The Author(s); Published by Published by Society of Diabetic Nephropathy Prevention. This is an open-access article distributed under the terms of the Creative Commons Attribution License (http://creativecommons.org/licenses/by/4.0), which permits unrestricted use, distribution, and reproduction in any medium, provided the original work is properly cited. 